\title{
7.432 HIDROTUBACIONES HISTERO-TUBARICAS EN EL CENTRO PERUANO DE FERTILIDAD MATRIMONIAL
}

\author{
Dr. Jorge Ascenzo Cabello 1 \\ Dr. Javier Hoyle Cox ${ }^{2}$ \\ Dr. Rafael de la Puente Lanfranco ${ }^{3}$ \\ Dr. Roberto Ruiz González ${ }^{4}$ \\ Dr. Heli Cancino Izaguirre ${ }^{5}$ \\ Dr. Alberto Ascenzo Palacio ${ }^{6}$
}

Nuestra experiencia sobre hidrotubaciones nos ha permitido, en el curso de once años, elaborar siete trabajos sobre esta clase de tratamiento médico del Factor Tubario alterado.

En nuestro último trabajo titulado “Hidrotubaciones Hístero Tubáricas en el Tratamiento de la Oclusión Distal Tubaria", Tema Oficial del $\vee$ Congreso Mundial de Fertilidad y Esterilidad, Estocolmo, Junio de 1966, decíamos: "Nuestros primeros resultados aparecen con 100 hidrotubaciones en Lima hace casi nueve años; luego con 450 hidrotubaciones se exponen, en junio de 1958, en el III Congreso Latino-Americano de Obstetricia y Ginecología, en México; y más tarde en Montreal en el II Congreso Mundial de la Federación Internacional de Ginecología y Obstetricia. Posteriormente, en junio de 1959, en Amsterdam, con motivo del III Congreso Mundial de Fertilidad y Esterilidad de la Asociación Internacional de Fertilidad, con 750 hidrotubaciones. Luego en Miami Beach, en enero de 1960, en la Conferencia
Panamericana de Infertilidad, sobre 1.000 hidrotubaciones $y$, por último, en la ciudad de Viena, en el III Congreso Mundial de la Federación Internacional de Ginecología y Obstetricia, en septiembre de 1961, con 1.700 hidrotubaciones. Un silencio de cinco años y una intensa actividad al respecto nos permiten presentar en la actualidad 5.560 hidrotubaciones practicadas a 602 estériles peruanas".

Hoy día, una experiencia sobre más de siete mil hidrotubaciones nos permite reafirmar nuestros conceptos, al ratificar la bondad de este tratamiento médico el cual rinde ampliamente si se capta en forma precisa hasta donde es posible buscar

1 Secretario General de la Asociación Internacional de Fertilidad.

2 Sub-Director del Centro Peruano de Fertilidad Matrimonial.

3 Delegado Oficial para Perú, de la Asociación Internacional de Fertilidad.

4 Médico especialista del Staff permanente del Centro Peruano de Fertilidad Matrimonial.

5 Vice-Presidente de la Sociedad Peruana de Fertilidad Matrimonial.

6 Médico especialista del Staff permanente del Centro Peruano de Fertilidad Matrimonial. 
sus beneficios, qué limitaciones tiene y sobre todo, en cuáles pacientes está indicado y en qué otras solo se logrará una pérdida de tiempo. Señalamos también, que un eventual tratamiento quirúrgico es favorecido por hidrotubaciones previas.

\section{Método}

Antes de iniciar las hidrotubaciones, practicamos una histerosalpingografía y dos persuflaciones con CO2, la segunda con antiespasmódico previo para evitar un error de diagnóstico.

Deben realizarse, de preferencia, en el período proliferativo; en general las iniciamos a partir del 7ํ. ú 8 ㅇ día del ciclo, a razón de tres por semana.

Efectuamos 1, 2 o 3 series de 10 hidrotubaciones cada una, según los resultados obtenidos que verificamos con el mismo procedimiento seguido antes de iniciar las hidrotubaciones.

Los resultados mejoran discretamente al aplicar, de preferencia en los mismos días, Ondas Cortas al hipogastrio y 15 ○ 20 mlgms. de algún derivado cortisónico por sesenta días. Actualmente las indicamos ocasionalmente.

\section{Técnica}

Es parecida a la de una histerosalpingografía; reemplazamos el opaco por una solución de cloranfenicol levógiro ( $1 / 2 \mathrm{gr}$.), fosfato de dexametazona ( 4 mlgms.) y/o hialuronidasa ( 250 unidades viscosimétricas) (x); complementamos a 20-30 ml. con suero fisiológico.

Un detalle de real interés es esperar $2 \circ 3$ minutos después del pasaje de los primeros centímetros cúbicos al interior del útero, hasta que ceda el espasmo en cuernos como respuesta fisiológica de la musculatura tubo-uterina a las maniobras instrumentales y al pasaje del líquido.

Es importante saber que la aplicación del medicamento debe ser a muy baja presión, pues no es la fuerza que se ejerce sobre el émbolo la que va a realizar el beneficio, sino la presión suave y sostenida que pone en contacto el líquido inyectado con la región que se trata de curar. Muchos fallan con hidrotubaciones solo por esto, pues a mayor fuerza mayor espasmo y dolor. La utilización de las cánulas desarrolladas por Fikentscher y Semm nos ha dado buenos resultado por lo que actualmente las empleamos con frecuencia.

\section{Contraindicaciones}

Es fundamental recordar que deben ser proscritas cuando existe un proceso infeccioso activo del tracto genital, así como si fluye sangre del interior del útero; por lo demás, tienen todas las contraindicaciones de una histerosalpingografía o persuflación.

\section{Material}

Hemos practicado 7.432 hidrotubaciones hístero-tubáricas a 775 pacientes, de las cuales 396 tenían franca obstrucción (51.0\%) y 379 comprobada estenosis (48.9\%).

La mayoría de las pacientes ha recibido 10 hidrotubaciones, pero, a pesar de haberse practicado hasta 20

(x) Nosotros utilizamos quemicetina succinato "Erba", $1 \mathrm{gm}$., Decadrón Mercb Sharp \& Dohme 1 frasco, $2 \mathrm{ml}$. (4 mlgms. por ml.) Wydase "Wyeth" 1 frasco de 500 unidades viscosimétricas. Empleamos la mitad de cada frasco por hidrotubación. 


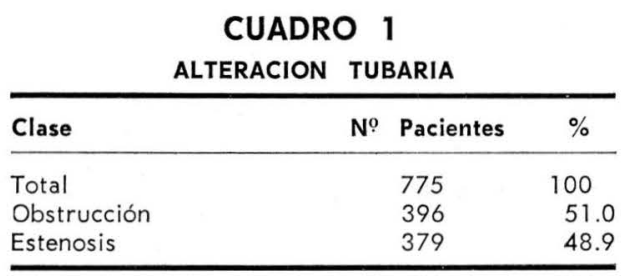

\section{GRAFICO N21}

ALTERACION TUBARIA

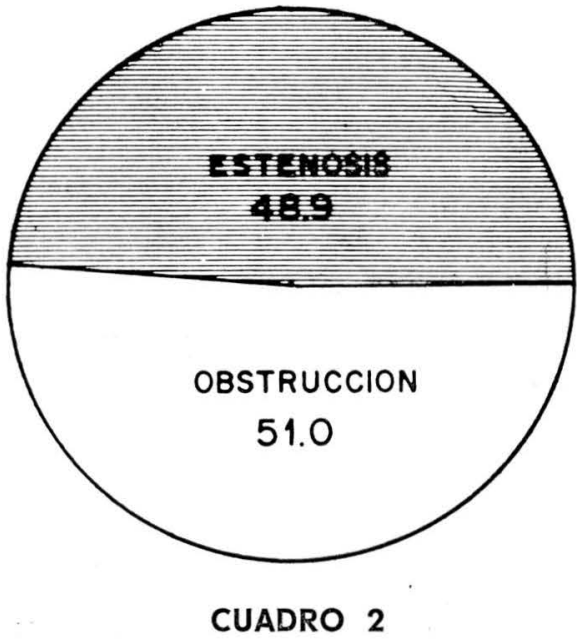

FRECUENCIA MEDIA DE HIDROTUBACIONES POR PACIENTE

\begin{tabular}{lr}
\hline Total hidrotubaciones: & 7.432 \\
Total pacientes: & 775 \\
Media: & 9.5 \\
\hline
\end{tabular}

e incluso hasta 30 , el promedio es ligeramente inferior a 10, dado a que algunos casos no han continuado las consultas.

En efecto, 7.432 hidrotubaciones realizadas a 775 estériles nos dan un promedio de 9.5 hidrotubaciones por paciente.

\section{Resultados}

Hemos logrado efectuar un control final a 568 mujeres; descartamos 207 casos por haber abandonado el estudio, por estar aún en tratamiento o por no haber regresado a su último control.

Nuestros resultados en estas 568 pacientes son los siguientes:

Observando el Cuadro 3 vemos que habian 171 estenosis y 75 obstrucciones entre las pacientes que normalizaron su Factor Tubario, así como 76 estenosis y 71 obstrucciones en las que mejoraron dicho factor y 32 estenosis y 144 obstrucciones entre las que fracasaron.

El Gráfico 3 expresa claramente lo anterior y con él podemos concluir fácilmente, que entre las que normalizaron hay menos de la mitad de obstrucciones que estenosis y entre las que fracasaron hay cerca de cinco veces más obstrucciones que estenosis.

CUADRO 3

7.432 HIDROTUBACIONES HISTERO-TUBARICAS EN ESTERILES PERUANAS

\begin{tabular}{|c|c|c|c|c|c|c|c|}
\hline Clasificación & $\begin{array}{c}\text { Total } p \\
N^{0}\end{array}$ & $\begin{array}{c}\text { entes } \\
\%\end{array}$ & Sub-Total & Cuernos & $\begin{array}{l}\text { Obstrucció } \\
1 / 3 \text { Medio }\end{array}$ & Pabellones & Estenosis \\
\hline Total & 568 & 100 & 289 & 98 & 62 & 129 & 279 \\
\hline Normalizaron & 246 & 433 & 75 & 17 & 13 & 45 & 171 \\
\hline Mejoraron & 146 & 257 & 70 & 15 & 16 & 39 & 76 \\
\hline Fracasaron & 176 & 309 & 144 & 66 & 33 & 45 & 32 \\
\hline
\end{tabular}




\section{GRAFICO Ne 3}

\section{COMPARACION DE LOS RESULTADOS ENTRE OBSTRUCCION $Y$ ESTENOSIS *}

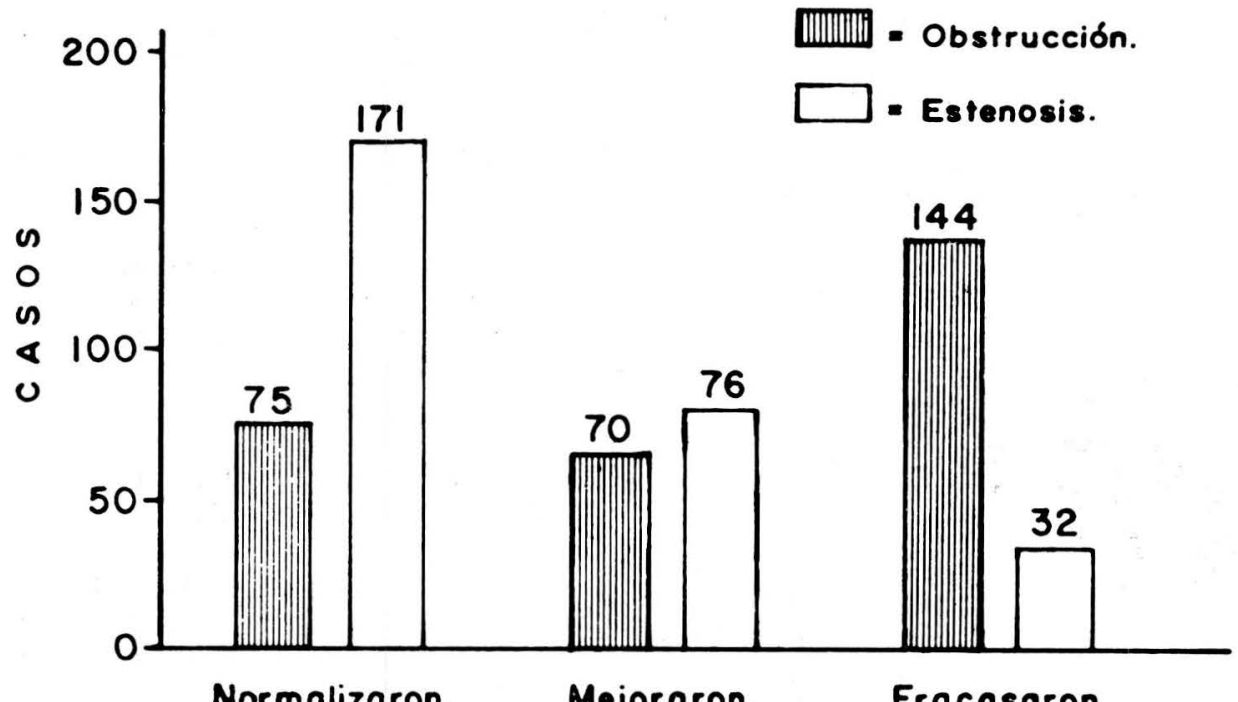

Normalizaron. Mejoraron. Fracasaron.

\section{\# 568 pacientes}

El Cuadro 3 nos muestra, también, los resultados según la localización de la obstrucción; para una mejor comprensión de éste hemos elaborado el Gráfico 3-A que nos permite objetivar claramente que de 98 pacientes con obstrucción en cuernos, normalizan 17 (relación 6 a 1); de 62 pacientes con obstrucción en tercio medio, normalizan 13 (relación 1 a 1 ); de 129 pacientes con obstrucción en pabellones, normalizan 45 (relación 3 a 1); de aquí concluímos que el pronóstico es peor cuanto más proximal es la obstrucción.

El tratamiento condujo a la normalización del Factor Tubario a 246

pacientes ( $43.3 \%$ del total de casos con control final); de éstas, 125 ( $50.8 \%$ de las que normalizaron) entraron en gestación y 121 (49.1\%) aunque normalizaron dicho Factor no lograron gestación por existir otros factores de esterilidad concomitantes (Cuadro 3-A).

Para obtener una visión de conjunto y práctica, juntamos los casos anteriores con los que mejoraron $y$ fracasaron, obteniendo el Cuadro 4 y el Gráfico 4.

Si agregamos a las que normalizaron, las pacientes que mejoraron, po- 
GRAFICO N:3·A

RESULTAdOS SEgUN LA LOCALIZACION DE LA OBSTRUCCION.*

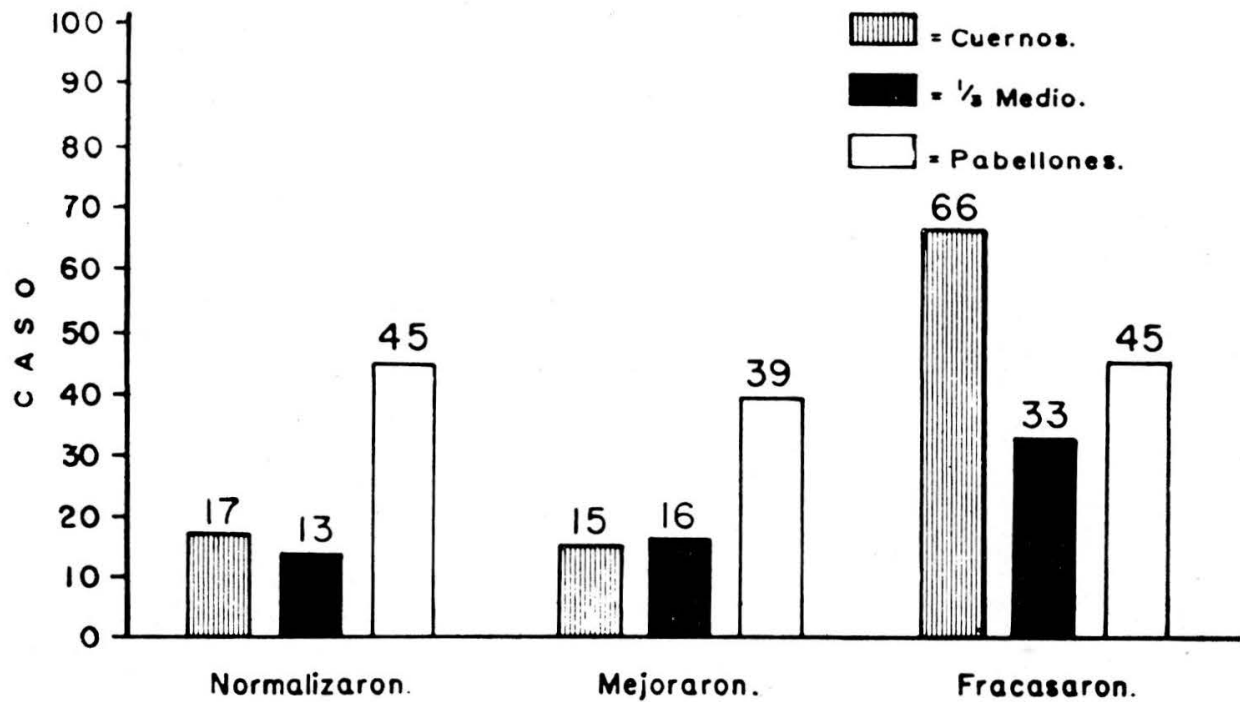

289 pacientes

CUADRO 3-A

PACIENTES QUE NORMALIZARON EL FACTOR TUBARIO POST-TRATAMIENTO

\begin{tabular}{|c|c|c|c|c|c|c|c|}
\hline Clsificación & $\begin{array}{c}\text { Total } \\
\mathbf{N}^{0}\end{array}$ & $\begin{array}{c}\text { ientes } \\
\%\end{array}$ & Sub-Total & Cuernos & $\begin{array}{l}\text { Obstrucción } \\
1 / 3 \text { Medio }\end{array}$ & Pabellones & Estenosis \\
\hline Total & 246 & 100 & 75 & 17 & 13 & 45 & 171 \\
\hline Gestaron & 125 & 50,8 & 41 & 7 & 8 & 26 & 84 \\
\hline No gestaron & 121 & 49,1 & 34 & 10 & 5 & 19 & 87 \\
\hline
\end{tabular}

CUADRO 4

ESTERILES CON CONTROL FINAL

\begin{tabular}{|c|c|c|c|}
\hline Resultados & $\mathbf{N}^{\mathbf{9}}$ & Pacientes & $\%$ \\
\hline $\begin{array}{l}\text { Total } \\
\text { Gestaciones } \\
\text { Normalizaron }\end{array}$ & & $\begin{array}{l}568 \\
125\end{array}$ & $\begin{array}{l}100 \\
22.0 *\end{array}$ \\
\hline sin gestación & & 121 & $21.3^{*}$ \\
\hline Mejoraron & & 146 & 25.7 \\
\hline Fracasaron & & 176 & 30.9 \\
\hline
\end{tabular}

* $43.3 \%$ de normalización, con y sin gestación (246 pacientes). demos concluir que las hidrotubaciones dan un 69.0 por ciento de resultados satisfactorios, como puede apreciarse en el Gráfico 4-A.

Nuestra experiencia sobre más de siete mil hidrotubaciones, nos hace ver la importancia que tienen las estenosis, las que generalmente son distales, a veces debidas a Factor Peritoneal y que impiden la gestación como si se tratara de una verdadera oclusión. 


\section{GRAFICO N24}

ESTERILES CON CONTROL FINAL

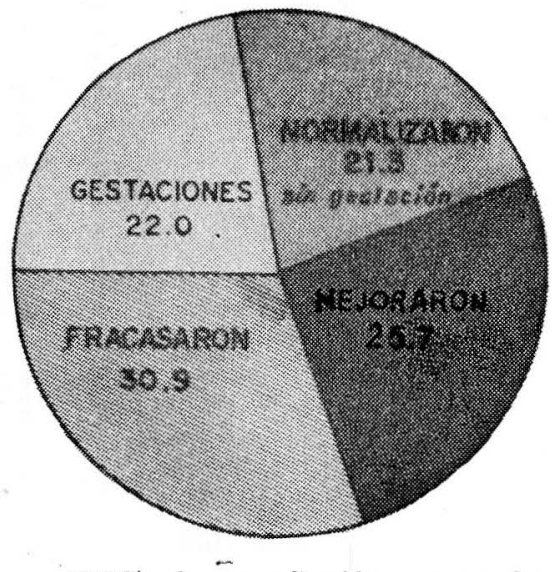

$43.3 \%$ de normalización, con y sin gestación (246 pacientes).

\section{GRAFICO N24A}

\section{ESTERILES CON CONTROL FINAL}

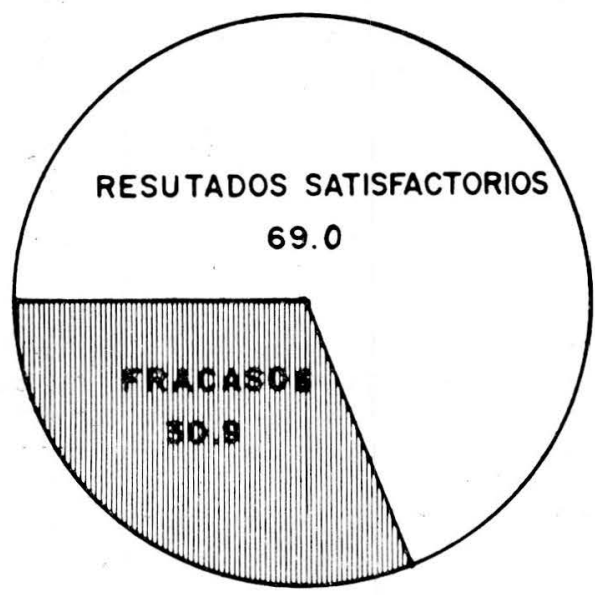

Estas estenosis toman una jerarquía formidable en el tratamiento de la mujer estéril por Factor Tubario alterado si éste se trata con hidrotubaciones; por eso hoy día, en mujeres con trompas en las que un estudio estático (histerosalpingografía) o dinámico (persuflación con anhídrido carbónico) no constatan absoluta normalidad, indicamos hidrotubaciones; es en este punto donde está lo trascendente de nuestro mensaje a los médicos que se inician en hidrotubaciones, pues es en estos casos donde la gestación recompensa con frecuencia este importante conocimiento; por supuesto ello exige una perfecta interpretación de las histerosalpingografías y de las curvas de persuflación de gases con anhídrido carbónico que son las que en realidad nos indicarán si existe o no la absoluta normalidad a que hacíamos referencia.

Nuestro Cuadro Estadístico 5 deja ver claramente que si nos hubiéramos limitado a practicar hidrotubaciones en los casos de oclusión distal tubárica, que es su indicación clásica, hubiéramos obtenido únicamente 45 casos de normalización del Factor Tubario en lugar de las 246 pacientes que lo normalizaron.

\section{CUADRO 5}

COMPARACION ENTRE NORMALIZACION EN OBSTRUCCION DISTAL, OTRAS LOCALIZACIONES DE LA OBSTRUCCION Y ESTENOSIS

\begin{tabular}{lcc}
\hline Clasificación & Pacientes & $\%$ \\
\hline Total & 246 & 100 \\
Obstrucción distal & 45 & 18.2 \\
Otras obstrucciones & 30 & 12.1 \\
Estenosis & 171 & 69.5 \\
\hline
\end{tabular}

Si expresamos las cifras anteriores en un gráfico, vemos claramente que hubiéramos desperdiciado 4/5 partes de nuestras posibilidades de éxito 
(Gráfico 5). La creencia de que las hidrotubaciones solo sirven para modificar obstrucciones del extremo externo de la trompa está tan difundida, que para el $\vee$ Congreso Mundial de Fertilidad y Esterilidad se nos pidió que hiciéramos un trabajo sobre el resultado de las hidrotubaciones en la oclusión distal tubaria.

\section{GRAFICO $N^{0} 5$}

Comparación entre normalización en obstrucción distal, otras localizaciones de la obstrucción y estenosis.

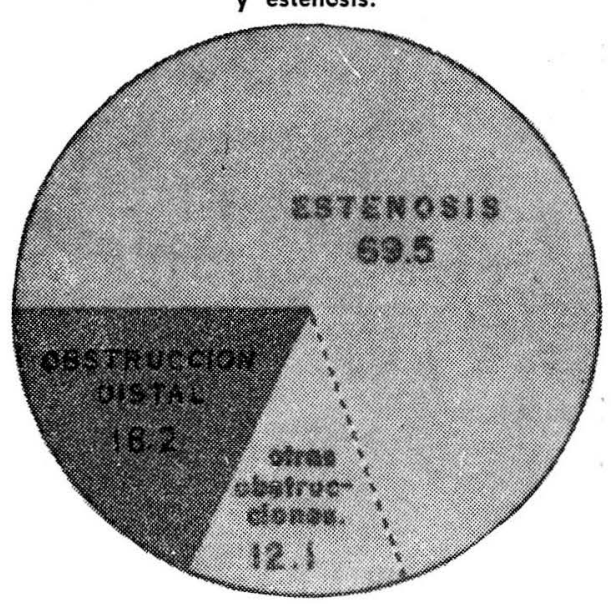

Refiriéndonos ahora solo a las obstrucciones, observamos que cuando las pacientes entraron en gestación hay casi cuatro veces más obstrucciones en el pabellón que en el cuerno (relación 4 a 1) (Cuadro 6). En cambio, en las que fracasaron se observa un menor número de obstrucciones en pabellones que en cuernos (relación 1 a 11/2) (Gráfico 6).

\section{CUADRO 6}

CUADRO COMPARATIVO DEL LUGAR DE LA OBSTRUCCION EN GESTACIONES Y FRACASOS

\begin{tabular}{lcc}
\hline Clasificación & Pabellón & Cuernos \\
\hline Total & 71 & 73 \\
Gestaron & 26 & 7 \\
Fracasaron & 45 & 66 \\
\hline
\end{tabular}

GRAFICO N 6

GRAFICO COMPARATIVO DEL LUGAR DE LA OBSTRUCCION EN GESTACIONES $Y$

FRACASOS.

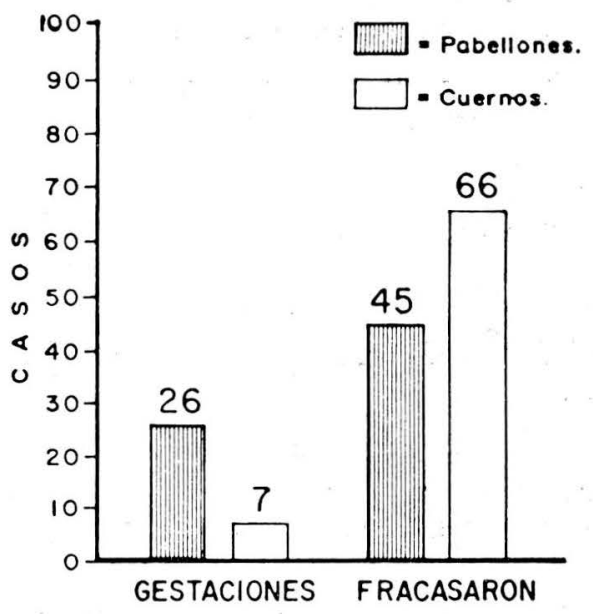

Los resultados obtenidos con las hidrotubaciones varían también según la etiología de la obstrucción o estenosis; así vemos que cuando las trompas se reobstruyen después de un tiempo de realizada una plastía tubaria, o cuancio la intervención quirúrgica no ha logrado permeabilizar las trompas, las hidrotubaciones siempre han fracasado. Este grueso estudio estadístico nos permite, por consiguiente, contraindicar las hidrotubaciones en los casos de plastía tubaria previa reobstruída; en cambio, es de todos conocida su bondad terapéutica cuando se inician inmediatamente después de la plastía quirúrgica, para conservar la permeabilidad obtenida con el acto operatorio.

De las 2.550 pacientes estériles estudiadas hasta el momento de hacer este trabajo, hemos encontrado 203 con TBC genital; de éstas seleccionamos 42 para ser hidrotubadas por 
tener el Factor Tubario menos comprometido, con lo que obtuvimos 4 gestaciones, todas hicieron embarazo tubario, 3 abortaron al Douglas y en la cuarta se encontró el huevo en el pabellón de la trompa izquierda, en una hubo gravísima hemorragia intraabdominal pre-operatoria.

Tanto en las que gestaron como en las que fracasaron observamos que el origen infeccioso o el antecedente de una operación ginecológica tenían un porcentaje similar; en cambio, los abortos criminales dentro de la etiología infecciosa llegaron al 16 por ciento entre las que gestaron y a un 54 por ciento entre las que fracasaron.

Se constató un 65 por ciento de antecedentes de metroanexitis entre la etiología infecciosa de las que gestaron.

Se advirtió en los casos fracasados que un tercio de ellos correspondía a trompas que habían tenido plastía tubaria y que luego se habían reobstruído.

\section{Complicaciones}

Hemos tenido 29 pacientes con complicaciones, de éstas, 21 tuvieron metroanexitis aguda $\circ$ sub-aguda $(2.7 \%)$; todas cedieron a tratamiento médico, solo una requirió hospitalización, no siendo en realidad severa en ningún caso.

En las pacientes con antecedentes de metroanexitis inyectamos profilácticamente, por vía parenteral, un antibiótico de amplio espectro después de cada hidrotubación.

Hubo 6 casos de embarazo ectópico $(0.7 \%)$.

Un estallido de trompa, sin complicaciones, producido en uno de los casos de plastía tubaria previa y un caso de shock de mediana intensidad, sumados a las complicaciones anteriores hacen el total de las 29 que nos dan un 3.6 por ciento de complicaciones (Cuadro 7 y Gráfico 7).

\section{CUADRO 7 \\ COMPLICACIONES POST-HIDROTUBACIONES}

\begin{tabular}{|c|c|c|c|}
\hline Clasificación & No & Pacientes & $\%$ \\
\hline Total & & 775 & 100 \\
\hline Metroanexitis aguda y & & & 27 \\
\hline $\begin{array}{l}\text { sub-aguda } \\
\text { Embarazo ectópico }\end{array}$ & & $\begin{array}{r}21 \\
6\end{array}$ & $\begin{array}{l}2.7 \\
0.7\end{array}$ \\
\hline Otras & & 2 & 0.2 \\
\hline Sin complicaciones & & 746 & 96.2 \\
\hline
\end{tabular}

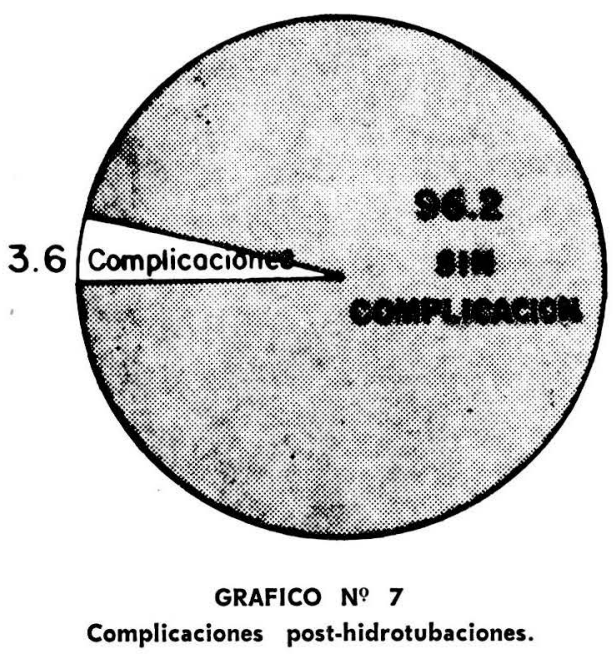

En las 7.432 hidrotubaciones practicadas, no hemos tenido ningún caso de muerte inmediata o mediata atribuíble a este tratamiento de la mujer estéril.

\section{Resumen y Conclusiones}

1. El trabajo simultáneo de seis especialistas del Centro Peruano de Fertilidad Matrimonial durante once años, nos ha permitido practicar 7.432 hidrotubaciones hístero tubári- 
cas a 775 estériles ( 9.5 hidrotubaciones por paciente).

En el transcurso de este tiempo hemos realizado siete trabajos al respecto.

2. Iniciamos las hidrotubaciones de preferencia a partir del $7^{\circ}$ บ $8^{\circ}$ día del ciclo, a razón de 3 por semana, efectuando 1, 2 ○ 3 series de 10 hidrotubaciones cada una, según los resultados obtenidos.

3. Tanto antes de iniciarlas como al terminar cada serie, practicamos una histerosalpingografía y dos persuflaciones con CO2, la segunda con antiespasmódico previo para evitar un error de diagnóstico.

4. Este tratamiento médico favorece una eventual plastía tubaria posterior.

5. Su técnica es parecida a la de una histerosalpingografía; reemplazamos el opaco por una solución de cloranfenicol levógiro ( $1 / 2 \mathrm{gr}$.) fosfato de dexametasona ( $4 \mathrm{mlgm}$.) $\mathrm{y} / \mathrm{o}$ hialuronidasa (250 unidades viscosimétricas); completamos a $20-30 \mathrm{ml}$. con suero fisiológico.

6. Sus contraindicaciones son las mismas que las que rigen para una histerosalpingografía o persuflación.

7. De las 775 pacientes con Factor Tubario alterado, 396 tenían franca obstrucción (51.0\%) y 379 comprobada estenosis $(48.9 \%)$ ).

8. Hemos logrado efectuar un control final a 568 pacientes; de éstas, en las que normalizaron hay menos de la mitad de obstrucciones que estenosis y entre las que fracasaron hay cerca de 5 veces más obstrucciones que estenosis.

9. El pronóstico es peor cuanto más proximal es la obstrucción tubaria.
10. Normalizaron 246 pacientes (43.3\% del total de casos con control final); 125 ( $50.8 \%$ de las que normalizaron) entraron en gestación y $121(49.1 \%)$ aunque normalizaron dicho Factor, no lograron gestación.

11. Las hidrotubaciones nos dan un 69.0 por ciento de resultados satisfactorios.

12. Si nos hubiéramos limitado a practicar hidrotubaciones solo en los casos de oclusión distal tubárica, donde su indicación es clásica, habríamos desperdiciado 4/5 partes de nuestras posibilidades de éxito.

13. El mensaje de este trabajo reside en indicarlas especialmente en las estenosis y en todo caso de trompas no enteramente normales a la histerosalpingografía o persuflación.

14. Cuando las pacientes entraron en gestación se comprobó cuatro veces más obstrucciones en el pabellón que en el cuerno; en cambio en las que fracasaron se observa un menor número de obstrucciones en pabellón que en cuernos.

15. Cuando las trompas se reobstruyen después de un tiempo de realizada una plastía tubaria o cuando la intervención quirúrgica no logra permeabilizar las trompas, las hidrotubaciones siempre fracasan.

16. Es probada su bondad terapéutica cuando se inician inmediatamente después de la plastía tubaria para conservar la permeabilidad obtenida con el acto quirúrgico.

17. Cuando las empleamos en casos seleccionados de TBC genital, por tener el Factor Tubario menos comprometido, obtuvimos el 10 por ciento de gestaciones, todas terminaron en embarazo ectópico. 
18. Los abortos criminales dentro de la etiología infecciosa llegaron a 16 por ciento entre las que gestaron y a 54 por ciento entre las que fracasaron.

19. Un tercio de los casos fracasados correspondía a trompas que habían tenido plastía tubaria y que luego se habían reobstruído.

20. Metroanexitis aguda o sub-aguda en 21 casos (solo una paciente requirió hospitalización, todas cedieron a tratamiento médico), seis embarazos ectópicos, un estallido de trompa (en un caso de plastía tubaria previa) y un caso de shock de mediana intensidad, nos dan un 3.6 por ciento de complicaciones para las hidrotubaciones.

21. De las 7.432 hidrotubaciones practicadas, no hemos tenido ningún caso de muerte inmediata o mediata atribuíble a este tratamiento.

\section{BIBLIOGRAFIA}

1 ALBANO V.; CITTADINI, E.; ROSSI, T.: World Congress on Fertility and Sterility, Proceedings, 1966, Excerpta Medica Foundation.

2 AMAYA L. M.: Rev. Colomb. Ginec. 1958, 9, 569

3 ARENAS N., BETTINOTTI A.: Esterilidad Conyugal. Editorial Bibliográfica Argentina, 1956.

4 ASCENZO-CABELLO, J., HOYLE C. J., DE LA PUENTE L. R., CHIONG CH. V., RUIZGONZALEZ R.: Estratto dall'Archivio de Ost. e Gin., 1958, 63, 485 Gin. y Obst. 1958, 6, 225. Gaceta Sanitaria, 1959, 13, 6.

5 ASCENZO-CABELLO J., HOYLE C. J., DE LA PUENTE, L. R., CHIONG CH. V., RUIZGONZALEZ R.: Intern. Journ. of Fertil. $1960,5,44$.

6 ASCENZO-CABELLO J., HOYLE C. J., DE LA PUENTE L. R., RUIZ-GONZALEZ, R. GAMARRA y E. B.: Ginec. y Obst. 1960, 6, 192.

7 ASCENZO-CABELLO J., HOYLE C. J., DE LA PUENTE L. R., RUIZ-GONZALEZ, R., GAMARRA y E. B.: III Congr. Intern. de Obst. y Gin., Vienna, Ag. 1961, Tomo I, pág. 205.
8 ASCENZO-CABELLO J. et al.: Intern. Journal of Fertility 1961, 6, 149.

9 ASCENZO-CABELLO J., HOYLE C. J., DE LA PUENTE R., RUIZ-GONZALEZ, R., CANCINO I. N.: $V$ World Congress on Fertility and Sterility, Proceedings, 1966, Excerpta Medica Foundation.

10 BEARZI L., GOLIGOW B., ESANDI J. J.: Semana Médica. 1961, 119, 81.

11 BENAVENTE LOZADA B., FERREYROS FALEN G.: Obst. Gin. Latin-Amer. 1961, 19, 581.

12 BOTELLA LLUSIA J.: Acta Ginecológica, XVII-5, 1966.

13 BRET H. J., LEGROS R.: Presse Med. 1956, 64, 2197.

14 BRNOUIN J. R.: C. R. Soc. Fr. Gyn. 1956, 26, 80.

15 BRUNO R. O.: Ginec. Latin. Amer., 1953, II, 55.

16 BUNSTER M. E.: Trompa de Falopio y Esterilidad de Causa Femenina, G. Kraft Ltda., Buenos Aires, pág. 305, 1951.

17 CAMACHO D. E.: La Prensa Médica Argentina, 46-252-1959.

18 CAMPOS DA PAZ FILHO A.: V World Congress on Fertility and Sterility Proceedings, 1966, Excerpta Medica Foundation.

19 CUSI M., URGELL J. M.: Rev. Española de Obst. y Ginec. 16-217-1959.

20 CUSI M., URGELL J.: Acta Ginecológica, II-103-1960.

21 CHASE S, V.: Primeras Jorn. Paraguayas de Fertil., 116-1961.

22 DE AQUINO SALLES A., PEANO M., DE MORALES FILHO, A., PREANO N.: $V$ World Congress on Fertility and Sterility, Proceedings, 1966, Excerpta Medica Foundation.

23 DE LA PEÑA REGIDOR P.: V World Congress on Fertility and Sterility, Proceedings, 1966, Excerpta Medica Foundation.

24 DO AMARAL FERREIRA C.: Anais Bras. de Gin. 43-185-1957.

25 DO AMARAL FERREIRA C., ALDELA $\vee$. B.: Anais Bras. de Gin. 48-289-1959.

26 DOUAY E.: Ginec. et Obst. 16-126-1927.

27 DRAGULESCU C. PAUL: Anal. de la Univ. Cent. de Venezuela, XLI-1956.

28 ELIAS COSTA J. D., GUGLIELMONE P.: Obst. y Gin. Latin.-Amer. 14-21-1960.

29 FIKENTSCHER R. and SEMM: Intern. Journ. of Fertility, 9-315-1964.

30 GAIGE JOSE E.: "Las Hidrotubaciones como Tratamiento de Esterilidad Post-Aborto". Trabajo de Incorporación a la Sociedad Peruana de Fertilidad Matrimonial. Sesión del 19 de Octubre de 1967.

31 GREEN ARMYTAGE V. B.: Brit. M. J. I1222-1959.

32 GRUBER A. E.: Gebursthilfe und Fravenheiblende, 19-72-1959. 
33 HYDE PRATT J., VANNER E. A., HUANG M.: Amer. J. Obst. and Gynec. 71-10971956.

34 INNERFIELD I.: Intern. Rec. Med. and GinPract. Clin., 168-687-1955.

35 INZA R.: Esterilidad Conyugal, Edit. Med. Panamericana, Buenos Aires.

36 ISRAEL LEON: "Diagnosis and Treatment of Menstrual Disorders and Sterility". Hoeber Medical Division, 442-469, 1967.

37 KUPMAN A.: Obst. Gin. Latin-Amer. 1584-1957.

38 LATORRETA G., NAPOLITANO M.: Archivo di Obst. e Ginec. 64-264-1959.

39 LAUFMAN H. y col.: Arch. Surg. 661-5221953.

40 LCURUS N., KASKARELIS D.: Rev. Franc. Gynec. Obst. 55-345-1960.

41 MARTIN G. J. y col.: Proc. Soc. Exper. Bid. Méd. 66-636-1954.

42 MARTIN G.: Min. Pinec. II-103-1959.

43 MORALES A., PEANO M.: Gynec. et Obst. 57-1244-1958.

44 MULHRAD S. J.: Presse Med. 67-1959.

45 MURRAY E., FOIX A.: Obst. Gine. LatinAmer. 15-84-1957.

46 NURBERGER L.: Znetblat f. Gynec. 46-491922.

47 PALMER R., MM. PALMER E.: Les Explorations Functionalles Gynecologiques. Masson et Cie. Paris. 267-324-1963.
48 PEÑA M. J., INZA R., USUBIAGA I., VIVOLI C.: Prensa Méd. Argentina. 46-26791959.

49 PEÑA H. J., INZA R., CONSOLI F.: Obst. Gin. Lat.-Amer. 17-508-1959.

50 PEÑA H. J., INZA R., CONSOLI F.: Sinop. Obst. Ginec. 7-515-1960.

51 PERALTA RAMOS A., PARADELO C.: Obst. y Gin. Lat-Amer. 5-6-16-224-1958.

52 RAMOS A. V., PEANO M.: An. Bras. Gin. 41-389-1956.

53 RUBIN I. C.: Uterotubal Insufflation. St. Louis. The C. V. Mosby Co. 1947.

54 RUEDA G. R.: Rev. Colomb. de Obst. y Gin. 8-115-1957.

55 SALVATIERRA V.: Rev. Esp. Obst. Ginec. 19-151-1960.

56 SANCHEZ G. A.: Estudios sobre Esterilidad. 12-57-1961.

57 SEREA G. E., VALERINO V., CHISALE E.: Min. Gin. 12-57-1961.

58 SPECK G.: Am. J. Obst. and Gin. 55-1048 1948.

59 VASPASIANO R. A., y PEANO M.: Anais Bras. de Gin. 41-389-1956.

60 WALTHER M. C.: Discussion from the paper from Ascenzo-Cabello J. y col.: Proceedings of the Panamerican Conference on Infertility of The I.F.A., Miami, 152-1960.

61 YAGI H.: Jap J. Obst. and Gin. 6-121929.

62 YAGI H.: Fertil. and Steril. 6-550-1955. 